\title{
SCIENTIFIC REPORTS

\section{The Association of Low Admission Serum Creatinine with the Risk of Respiratory Failure Requiring Mechanical Ventilation: A Retrospective Cohort Study}

\author{
Charat Thongprayoon ${ }^{1}$, Wisit Cheungpasitporn ${ }^{2}$, Api Chewcharat ${ }^{1}$, Michael A. Mao ${ }^{3}$, \\ Sorkko Thirunavukkarasu ${ }^{1} \&$ Kianoush B. Kashani ${ }^{1,4^{*}}$
}

To assess the association between low serum creatinine $(\mathrm{SCr})$ value at admission and the risk of respiratory failure requiring mechanical ventilation in hospitalized patients. A retrospective cohort study was conducted at a tertiary referral hospital. All hospitalized adult patients from 2011 through 2013 who had an admission SCr value were included in this study. Patients who were mechanically ventilated at the time of admission were excluded. Admission creatinine was stratified into 7 groups: $\leq 0.4,0.5-0.6,0.7-0.8,0.9-1.0,1.1-1.2,1.3-1.4$, and $\geq 1.5 \mathrm{mg} / \mathrm{dL}$. The primary outcome was the occurrence of respiratory failure requiring mechanical ventilation during hospitalization. Logistic regression analysis was used to assess the independent risk of respiratory failure based on various admission $\mathrm{SCr}$, using $\mathrm{SCr}$ of $0.7-0.8 \mathrm{mg} / \mathrm{dL}$ as the reference group in the analysis of all patients and female subgroup and of $0.9-1.0 \mathrm{mg} / \mathrm{dL}$ in analysis of male subgroup. A total of 67,045 eligible patients, with the mean admission $\mathrm{SCr}$ of $1.0 \pm 0.4 \mathrm{mg} / \mathrm{dL}$, were studied. Of these patients, 799 (1.1\%) had admission $\mathrm{SCr}$ of $\leq 0.4 \mathrm{mg} / \mathrm{dL}$, and $2886(4.3 \%)$ developed respiratory failure requiring mechanical ventilation during hospitalization. The U-curve relationship between admission $\mathrm{SCr}$ and respiratory failure during hospitalization was observed, with the nadir incidence of in-hospital respiratory failure in $\mathrm{SCr}$ of $0.7-0.8 \mathrm{mg} / \mathrm{dL}$ and increased in-hospital respiratory failure associated with both reduced and elevated admission SCr. After adjustment for confounders, very low admission $\mathrm{SCr}$ of $\leq 0.4 \mathrm{mg} /$ $\mathrm{dL}$ was significantly associated with increased in-hospital respiratory failure $(\mathrm{OR} 3.11 ; 95 \% \mathrm{Cl} 2.33-$ 4.17), exceeding the risk related to markedly elevated admission $\mathrm{SCr}$ of $\geq 1.5 \mathrm{mg} / \mathrm{dL}$ (OR $1.61 ; 95 \% \mathrm{Cl}$ 1.39-1.85). The association remained significant in the subgroup analysis of male and female patients. Low SCr value at admission is independently associated with increased in-hospital respiratory failure requiring mechanical ventilation in hospitalized patients.

Creatinine is a breakdown metabolite of creatine phosphate in muscle ${ }^{1}$. It is eliminated by kidneys, mostly via glomerular filtration and partly via secretion at proximal tubule ${ }^{1,2}$. Therefore, creatinine clearance could be used to estimate the glomerular filtration rate (eGFR) by measuring serum creatinine (SCr), urine creatinine, and volume $^{3,4}$. Additionally, SCr can be a reflection of muscle mass and diet. Some patients might have decreased SCr independent of eGFR such as vegetarians, patients with muscle wasting or critically ill individuals ${ }^{3,5-7}$.

Respiratory failure is a serious condition caused by either lung pathology resulting in imbalances in oxygen or carbon dioxide exchanges. Ventilation of air in the lungs closely depends on strength and workload of respiratory muscles ${ }^{8}$. Failure of ventilation is one of the major causes of acute respiratory failure. Management of respiratory

${ }^{1}$ Division of Nephrology and Hypertension, Department of Medicine, Mayo Clinic, Rochester, MN, USA. ${ }^{2}$ Division of Nephrology, Department of Internal Medicine, University of Mississippi Medical Center, Jackson, Mississippi, USA. ${ }^{3}$ Division of Nephrology and Hypertension, Mayo Clinic, Jacksonville, FL, 32224, USA. ${ }^{4}$ Division of Pulmonary and Critical Care Medicine, Department of Medicine, Mayo Clinic, Rochester, MN, USA. *email: kashani.kianoush@ mayo.edu 
failure includes treating the primary cause of respiratory failure and ventilatory support. The respiratory support could include nasal cannula, noninvasive ventilation, invasive ventilation, and ECMO ${ }^{9}$. In severe respiratory failure, to improve gas exchange and decrease work of breathing, mechanical ventilation is often needed ${ }^{10}$. Malnutrition, premorbid frailty, and severity of illness (measured by Acute Physiologic Assessment and Chronic Health Evaluation (APACHE) II score) are associated with a higher need of mechanical ventilation among respiratory failure patients. Even though low SCr is associated with poor nutritional status and decreased muscle mass, low SCr has not been reported in the literature to be a predictor of respiratory failure requiring mechanical ventilation.

Therefore, our goal is to investigate the association between low $\mathrm{SCr}$ at admission and the risk of respiratory failure requiring mechanical ventilation.

\section{Materials and Methods}

Study population. We conducted this retrospective cohort study at a tertiary referral center care. We explored our hospital database to collect data on all patients aged $\geq 18$ years and admitted to Mayo Clinic, Minnesota, between January $1^{\text {st }}, 2011$ and December 31 $1^{\text {st }}, 2013$, who had available SCr measurement at the time of admission. Patients who had end-stage renal disease or were on mechanical ventilation at hospital admission were excluded. ESRD was determined by International Classification of Diseases, 9th Revision, (ICD-9) diagnosis code of $458.21,585.5,585.6,792.5,996.56,996.68$, and 996.73 or by eGFR $<15 \mathrm{~mL} / \mathrm{min} / 1.73 \mathrm{~m}^{2}$. If a patient had recurring hospital admissions throughout the study period, we used only data on the first hospital admission. Mayo Clinic Institutional Review Board reviewed and approved this project (IRB number 15-000024) and waived informed consent due to the minimal risk nature of this study. The study was conducted in accordance with the relevant guidelines and regulations.

Data collection. We collected the needed data from our institutional electronic medical record system. This database comprises demographic characteristics, diagnosis and procedure codes, hospital admission information, flow sheet information of inpatients and outpatients, and results of laboratory test. We defined the admission SCr as the initial SCr level in 24 hours of hospital admission. We categorized admission SCr into 7 groups: $\leq 0.4$, $0.5-0.6,0.7-0.8,0.9-1.0,1.1-1.2,1.3-1.4$ and $\geq 1.5 \mathrm{mg} / \mathrm{dL}$. We calculated body mass index (BMI) using (weight in $\mathrm{kg} /[\text { height in } \mathrm{m}]^{2}$ ) at admission. We grouped principal diagnoses based on ICD-9 code. We collected comorbid condition utilizing a previously validated information abstraction algorithm and calculated the Charlson Comorbidity Index $(\mathrm{CCI})^{11}$ to evaluate comorbidity burden.

Clinical outcomes. The outcome of interest was respiratory failure requiring mechanical ventilation during hospitalization. We did not include the elective use of mechanical ventilation during procedure or operation as the outcome. We also assessed the duration of mechanical ventilation among patients with RF requiring mechanical ventilation.

Statistical analysis. We summarize continuous variables as mean \pm standard deviation (SD) and categorical variables as number (\%). We compared baseline demographics and clinical characteristics between admission SCr groups utilizing the $\chi^{2}$ test for categorical variables and analysis of variance (ANOVA) for continuous variables. The restricted cubic spline, with six knots were placed at $0.4,0.6,0.8,1.0,1.2$ and $1.4 \mathrm{mg} / \mathrm{dL}$ of serum creatinine, was utilized to depict the potential non-linear association between SCr at the admission and risk of respiratory failure requiring mechanical ventilation. In the analysis of all patients, an admission SCr group of $0.7-0.8 \mathrm{mg} / \mathrm{dL}$ was used as the reference group for comparison. For the subgroup analyses, we use the same SCr range for female patients, whereas we selected an admission SCr group of $0.9-1.0 \mathrm{mg} / \mathrm{dL}$ in the analysis of male patients. These SCr limits were associated with the lowest unadjusted in-hospital mortality. We performed logistic regression analysis to assess the independent association of SCr levels with the development of RF requiring mechanical ventilation during hospitalization. We constructed a multivariable model to adjust for priori-defined covariates, including age, race, sex, BMI, principal diagnosis, CCI, diabetes mellitus, congestive heart failure, coronary artery disease, peripheral vascular disease, stroke, chronic obstructive pulmonary disease, hemiplegia/paraplegia, or cirrhosis. We performed subgroup analysis stratified by sex and tested interaction between sex and admission SCr on respiratory failure outcome. We performed linear regression analysis to assess the independent association between admission SCr levels and mechanical ventilation duration among patients with respiratory failure. As mechanical ventilation duration had a skewed distribution, hence, we summarized duration as median with interquartile range (IQR) and we log-transformed its value before entering into the model (Fig. S1). We reported the relative prolongation, which was derived from exponential of the regression coefficient. We considered a 2 -tailed $P$ value $<0.05$ statistically significant. When not specified, we performed all analyses using JMP statistical software Version 10 (SAS Institute Inc., USA). We constructed restricted cubic spline using STATA version 14.1 (StataCorp LLC, Texas, USA).

Institution. This work was performed at Mayo Clinic in Rochester, MN.

\section{Results}

Baseline characteristics. Among 76,696 hospitalized patients with available SCr at hospital admission through the study period, we excluded 2,702 ESRD patients and 6,949 patients who were on mechanical ventilation at hospital admission to include a total of 67,045 patients in the analysis. Table 1 describes the clinical characteristics. The mean age was $61 \pm 18$ years. Fifty-two percent of the enrolled patients were men. The mean BMI and admission SCr were $29.8 \pm 7.7 \mathrm{~kg} / \mathrm{m}^{2}$ and $1.0 \pm 0.4 \mathrm{mg} / \mathrm{dL}$, respectively. Very low admission $\mathrm{SCr}$ of $\leq 0.4 \mathrm{mg} / \mathrm{dL}$ was observed in $779(1.2 \%)$ patients, whereas 6,174 (9.2\%) had markedly elevated admission SCr of $\geq 1.5 \mathrm{mg} / \mathrm{dL}$. Patients with low admission SCr tended to be younger, female, non-White, with lower BMI, and albumin while 


\begin{tabular}{|c|c|c|c|c|c|c|c|c|c|c|}
\hline \multirow[b]{2}{*}{ Variables } & \multirow[b]{2}{*}{ All } & \multicolumn{9}{|c|}{ Serum creatinine level at hospital admission (mg/dL) } \\
\hline & & $\leq 0.4$ & $0.5-0.6$ & $0.7-0.8$ & $0.9-1.0$ & $1.1-1.2$ & 1.3-1.4 & $\geq 1.5$ & $\mathbf{p}$ & $\begin{array}{l}\mathbf{p} \text {-for } \\
\text { trend }\end{array}$ \\
\hline $\mathrm{N}$ & 67045 & 799 & 9021 & 20422 & 17413 & 9122 & 4094 & 6174 & & \\
\hline Age, year & $61 \pm 18$ & $54 \pm 18$ & $55 \pm 18$ & $58 \pm 18$ & $61 \pm 17$ & $65 \pm 17$ & $69 \pm 15$ & $71 \pm 15$ & $<0.001$ & $<0.001$ \\
\hline Male & $34722(52)$ & $139(17)$ & $1451(16)$ & $7703(38)$ & $11553(66)$ & $6674(73)$ & $2879(70)$ & $4323(70)$ & $<0.001$ & $<0.001$ \\
\hline Caucasian & $62418(93)$ & $681(85)$ & $8150(90)$ & $19022(93)$ & $16320(94)$ & $8594(94)$ & $3852(94)$ & $5799(94)$ & $<0.001$ & $<0.001$ \\
\hline $\mathrm{BMI}, \mathrm{kg} / \mathrm{m}^{2}$ & $29.8 \pm 7.7$ & $25.8 \pm 7.8$ & $28.4 \pm 8.1$ & $29.5 \pm 7.8$ & $30.1 \pm 7.4$ & $30.3 \pm 7.2$ & $30.5 \pm 7.5$ & $30.8 \pm 7.9$ & $<0.001$ & $<0.001$ \\
\hline Principal Diagnosis & & & & & & & & & $<0.001$ & - \\
\hline Cardiovascular & $11653(17)$ & $57(7)$ & $864(10)$ & $2769(14)$ & $3160(18)$ & $2068(23)$ & $1028(25)$ & $1707(28)$ & & \\
\hline Endocrine/Metabolic & $1851(3)$ & $38(5)$ & $286(3)$ & $571(3)$ & $358(2)$ & $199(2)$ & $130(3)$ & $269(4)$ & & \\
\hline Gastrointestinal & $6651(10)$ & $100(13)$ & $1068(12)$ & $2185(11)$ & $1556(9)$ & $766(8)$ & $387(9)$ & $589(10)$ & & \\
\hline Hematology/Oncology & $11138(17)$ & $118(15)$ & $1522(17)$ & $3208(16)$ & $3039(17)$ & $1646(18)$ & $709(17)$ & $896(15)$ & & \\
\hline Infectious Disease & $1935(3)$ & $26(3)$ & $234(3)$ & $495(2)$ & $384(2)$ & $260(3)$ & $160(4)$ & $376(6)$ & & \\
\hline Respiratory & $2670(4)$ & $80(10)$ & $346(4)$ & $753(4)$ & $623(4)$ & $353(4)$ & $194(5)$ & $321(5)$ & & \\
\hline Injury/poisoning & $10539(16)$ & $142(18)$ & $1514(17)$ & $3344(16)$ & $2852(16)$ & $1418(16)$ & $563(14)$ & $706(11)$ & & \\
\hline Other & $20608(31)$ & $127(30)$ & $3187(35)$ & $7097(35)$ & $5441(31)$ & $2412(26)$ & $923(23)$ & $1310(21)$ & & \\
\hline Charlson score & $1.7 \pm 2.3$ & $1.9 \pm 2.5$ & $1.5 \pm 2.3$ & $1.4 \pm 2.1$ & $1.5 \pm 2.2$ & $1.9 \pm 2.3$ & $2.5 \pm 0.6$ & $3.0 \pm 2.7$ & $<0.001$ & $<0.001$ \\
\hline \multicolumn{11}{|l|}{ Comorbidities } \\
\hline CAD & $4738(7)$ & $29(4)$ & $273(3)$ & $950(5)$ & $1210(7)$ & $809(9)$ & $530(13)$ & $937(15)$ & $<0.001$ & $<0.001$ \\
\hline CHF & $4010(6)$ & $24(3)$ & $209(2)$ & $624(3)$ & $828(5)$ & $691(8)$ & $496(12)$ & $1138(18)$ & $<0.001$ & $<0.001$ \\
\hline PVD & $1839(3)$ & $14(2)$ & $121(1)$ & $341(2)$ & $376(2)$ & $310(3)$ & $211(5)$ & $466(8)$ & $<0.001$ & $<0.001$ \\
\hline Stroke & $4712(7)$ & $37(5)$ & $416(5)$ & $1076(5)$ & $1129(6)$ & $747(8)$ & $447(11)$ & $860(14)$ & $<0.001$ & $<0.001$ \\
\hline $\mathrm{DM}$ & 12457 (19) & $138(17)$ & $1297(14)$ & $2949(14)$ & $2824(16)$ & $1882(21)$ & $1107(27)$ & $2260(37)$ & $<0.001$ & $<0.001$ \\
\hline COPD & $5456(8)$ & $80(10)$ & $616(7)$ & $1287(6)$ & $1198(7)$ & $882(10)$ & $484(12)$ & $909(15)$ & $<0.001$ & $<0.001$ \\
\hline Cirrhosis & $1532(2)$ & $27(3)$ & $214(2)$ & $387(2)$ & $272(2)$ & $191(2)$ & $127(3)$ & $314(5)$ & $<0.001$ & $<0.001$ \\
\hline Hemi/paraplegia & $317(0.4)$ & $25(3)$ & $102(1)$ & $83(0.4)$ & $42(0.2)$ & $24(0.3)$ & $17(0.4)$ & $24(0.4)$ & $<0.001$ & $<0.001$ \\
\hline Albumin. $\mathrm{g} / \mathrm{dL}(\mathrm{n}=8733)$ & $3.6 \pm 0.7$ & $3.1 \pm 0.7$ & $3.4 \pm 0.7$ & $3.6 \pm 0.7$ & $3.7 \pm 0.7$ & $3.6 \pm 0.7$ & $3.5 \pm 0.7$ & $3.4 \pm 0.7$ & $<0.001$ & $<0.001$ \\
\hline
\end{tabular}

Table 1. Baseline clinical characteristics. Continuous data are presented as mean $\pm \mathrm{SD}$; categorical data are presented as count (\%). Abbreviations: BMI, body mass index; CAD, coronary artery disease; CHF, congestive heart failure; COPD, chronic obstructive pulmonary disease; DM, diabetes mellitus; PVD, peripheral vascular disease;

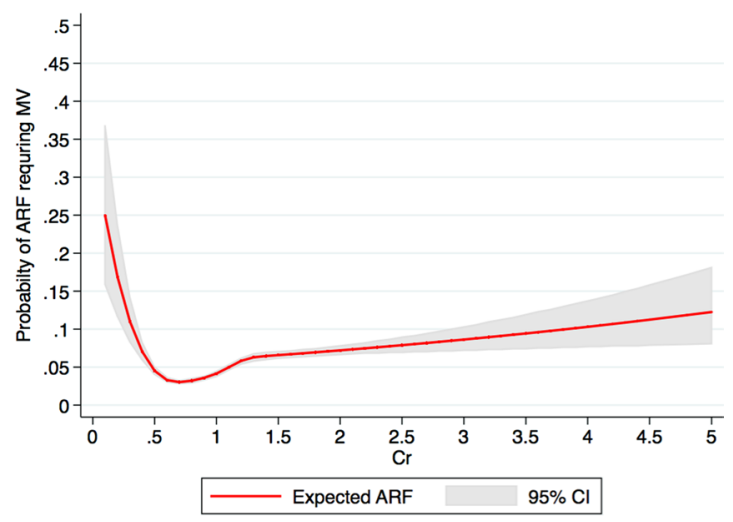

Figure 1. A restricted cubic spline showed the association between admission serum creatinine and risk of inhospital respiratory failure requiring mechanical ventilation. Figure was created using STATA (StataCorp. 2015. Stata Statistical Software: Release 14. College Station, TX: StataCorp LP).

they had a higher prevalence of hemiplegia/paraplegia. The association of various admission SCr levels with CCI revealed a U-shaped distribution, with a nadir of CCI at an admission SCr of $0.7-0.8 \mathrm{mg} / \mathrm{dL}(P<0.001)$.

Respiratory failure requiring mechanical ventilation. Among 67,045 patients, 2,886 (4.3\%) developed respiratory failure requiring mechanical ventilation during hospitalization. We found a U-shaped distribution for in-hospital respiratory failure and admission SCr levels, with the lowest in-hospital respiratory failure found in patients with admission SCr of $0.7-0.8 \mathrm{mg} / \mathrm{dL}$ (Fig. 1). When adjusted for age, race, sex, BMI, principal diagnosis, 


\begin{tabular}{|c|c|c|c|c|c|}
\hline \multirow{2}{*}{$\begin{array}{l}\text { Serum creatinine level at } \\
\text { hospital admission (mg/dl) }\end{array}$} & \multirow{2}{*}{$\begin{array}{l}\text { Mechanical ventilator } \\
\text { in hospital }\end{array}$} & \multicolumn{2}{|c|}{ Univariate analysis } & \multicolumn{2}{|l|}{ Multivariate analysis } \\
\hline & & OR (95\% CI) & $\mathbf{p}$ & Adjusted OR $(95 \% \mathrm{CI}) *$ & $\mathbf{P}$ \\
\hline$\leq 0.4$ & $69(8.6)$ & $2.87(2.22-3.72)$ & $<0.01$ & $3.11(2.33-4.17)$ & $<0.001$ \\
\hline $0.5-0.6$ & $315(3.5)$ & $1.10(0.96-1.26)$ & 0.17 & $1.29(1.11-1.50)$ & 0.001 \\
\hline $0.7-0.8$ & $650(3.2)$ & 1 (ref) & - & 1 (ref) & - \\
\hline $0.9-1.0$ & $670(3.9)$ & $1.22(1.09-1.36)$ & 0.001 & $1.07(0.95-1.21)$ & 0.23 \\
\hline $1.1-1.2$ & $472(5.2)$ & $1.66(1.47-1.87)$ & $<0.001$ & $1.30(1.13-1.49)$ & 0.001 \\
\hline $1.3-1.4$ & $250(6.1)$ & $1.98(1.70-2.30)$ & $<0.001$ & $1.45(1.23-1.71)$ & $<0.001$ \\
\hline$\geq 1.5$ & $460(7.5)$ & $2.45(2.17-2.77)$ & $<0.001$ & $1.61(1.39-1.85)$ & $<0.001$ \\
\hline
\end{tabular}

Table 2. The association between admission serum creatinine levels and in-hospital respiratory failure requiring mechanical ventilation. *Adjusted for age, sex, race, BMI, principal diagnosis, Charlson Comorbidity Index, coronary artery disease, congestive heart failure, peripheral vascular disease, stroke, diabetes mellitus, chronic obstructive pulmonary disease, cirrhosis, hemi/paraplegia.

\begin{tabular}{|c|c|c|c|c|c|}
\hline \multicolumn{6}{|l|}{ (a)Male $(\mathrm{n}=34722)$} \\
\hline \multirow[b]{2}{*}{$\begin{array}{l}\text { Serum creatinine level at } \\
\text { hospital admission }(\mathrm{mg} / \mathrm{dl})\end{array}$} & \multirow[b]{2}{*}{$\begin{array}{l}\text { Mechanical ventilator } \\
\text { in hospital }\end{array}$} & \multicolumn{2}{|c|}{ Univariate analysis } & \multicolumn{2}{|c|}{ Multivariate analysis } \\
\hline & & OR $(95 \% \mathrm{CI})$ & $\mathbf{p}$ & $\begin{array}{l}\text { Adjusted OR } \\
(95 \% \mathrm{CI})^{*}\end{array}$ & $\mathbf{P}$ \\
\hline$\leq 0.4$ & $15(10.8)$ & $2.83(1.64-4.87)$ & 0.001 & $3.35(1.79-6.26)$ & 0.001 \\
\hline $0.5-0.6$ & $79(5.4)$ & $1.34(1.05-1.72)$ & 0.02 & $1.46(1.12-1.90)$ & 0.007 \\
\hline \begin{tabular}{|l|}
$0.7-0.8$ \\
\end{tabular} & $326(4.2)$ & $1.03(0.89-1.19)$ & 0.66 & $1.04(0.89-1.21)$ & 0.63 \\
\hline $0.9-1.0$ & $474(4.1)$ & 1 (ref) & - & 1 (ref) & - \\
\hline $1.1-1.2$ & $339(5.1)$ & $1.25(1.08-1.44)$ & 0.002 & $1.12(0.96-1.30)$ & 0.16 \\
\hline $1.3-1.4$ & $180(6.3)$ & $1.56(1.31-1.86)$ & $<0.001$ & $1.31(1.08-1.58)$ & 0.006 \\
\hline$\geq 1.5$ & $324(7.5)$ & $1.89(1.64-2.19)$ & $<0.001$ & $1.40(1.19-1.65)$ & $<0.001$ \\
\hline \multicolumn{6}{|l|}{ (b) Female $(n=32323)$} \\
\hline$\leq 0.4$ & $54(8.2)$ & $3.41(2.52-4.60)$ & $<0.001$ & $3.20(2.29-4.47)$ & $<0.001$ \\
\hline $0.5-0.6$ & $236(3.1)$ & $1.23(1.04-1.46)$ & 0.02 & $1.32(1.10-1.58)$ & 0.003 \\
\hline \begin{tabular}{|l|}
$0.7-0.8$ \\
\end{tabular} & $324(2.6)$ & 1 (ref) & - & 1 (ref) & - \\
\hline $0.9-1.0$ & $196(3.3)$ & $1.32(1.11-1.59)$ & 0.003 & $1.21(0.99-1.47)$ & 0.06 \\
\hline $1.1-1.2$ & $133(5.4)$ & $2.20(1.79-2.70)$ & $<0.001$ & $1.81(1.45-2.26)$ & $<0.001$ \\
\hline $1.3-1.4$ & $70(5.8)$ & $2.34(1.79-3.05)$ & $<0.001$ & $1.78(1.33-2.37)$ & 0.001 \\
\hline$\geq 1.5$ & $136(7.4)$ & $3.03(2.47-3.73)$ & $<0.001$ & $2.12(1.68-2.67)$ & $<0.001$ \\
\hline
\end{tabular}

Table 3. The association between admission serum creatinine levels and in-hospital respiratory failure requiring mechanical ventilation stratified by sex Adjusted for age, race, BMI, principal diagnosis, Charlson Comorbidity Index, coronary artery disease, congestive heart failure, peripheral vascular disease, stroke, diabetes mellitus, chronic obstructive pulmonary disease, cirrhosis, hemi/paraplegia. $\mathrm{P}$ for interaction $=0.01$.

and comorbidities, low admission SCr of $\leq 0.4,0.5-0.6 \mathrm{mg} / \mathrm{dL}$, and elevated admission of 1.1-1.2, 1.3-1.4, and $\geq 1.5 \mathrm{mg} / \mathrm{dL}$ were significantly associated with increased in-hospital respiratory failure, compared with the admission SCr of $0.7-0.8 \mathrm{mg} / \mathrm{dL}$ (Table 2). The risk associated with very low SCr of $\leq 0.4 \mathrm{mg} / \mathrm{dL}$ surpassing the risk associated with high SCr of $\geq 1.5 \mathrm{mg} / \mathrm{dL}$ (OR $3.11 \mathrm{vs.} 1.61$, respectively).

Subgroup analysis of respiratory failure based on sex. Among 34,722 male patients, the mean admission SCr level was $1.1 \pm 0.4 \mathrm{mg} / \mathrm{dL}$. Very low admission SCr of $\leq 0.4$ noted in $139(0.4 \%)$ and $1,737(5.0 \%)$ developed respiratory failure requiring mechanical ventilation during hospitalization. The lowest incidence of in-hospital respiratory failure was in male patients with admission SCr of $0.9-1.0 \mathrm{mg} / \mathrm{dL}$ (4.1\%). In adjusted analysis, low admission SCr of $\leq 0.4,0.5-0.6 \mathrm{mg} / \mathrm{dL}$, and elevated admission $\mathrm{SCr}$ of $1.3-1.4$, and $\geq 1.5 \mathrm{mg} / \mathrm{dL}$ were significantly associated with higher risk of in-hospital respiratory failure compared with admission SCr of 0.9-1.0 mg/dL (Table 3a, Fig. 2).

Among 32,323 female patients, the mean admission SCr was $0.8 \pm 0.3 \mathrm{mg} / \mathrm{dL}$. Very low admission SCr $\leq 0.4$ was seen in $660(2.0 \%)$ patients, and 1,149 (3.6\%) developed respiratory failure requiring mechanical ventilation during hospitalization. The lowest incidence of in-hospital respiratory failure was found in female patients with admission SCr of $0.7-0.8 \mathrm{mg} / \mathrm{dL}(2.6 \%)$. In adjusted analysis, low admission $\mathrm{SCr}$ of $\leq 0.4,0.5-0.6 \mathrm{mg} / \mathrm{dL}$, and elevated admission SCr of 1.1-1.2, 1.3-1.4, and $\geq 1.5 \mathrm{mg} / \mathrm{dL}$ were significantly associated with increased risk of in-hospital respiratory failure compared with admission SCr of $0.7-0.8 \mathrm{mg} / \mathrm{dL}$ (Table 3b, Fig. 2). There was an interaction between sex and admission SCr on the risk of in-hospital respiratory failure ( $\mathrm{p}$ for interaction $=0.01$ ).

Duration of mechanical ventilation among respiratory failure patients. The median duration of mechanical ventilation was 46 (IQR 20-117) hours among patients with respiratory failure. A restricted 


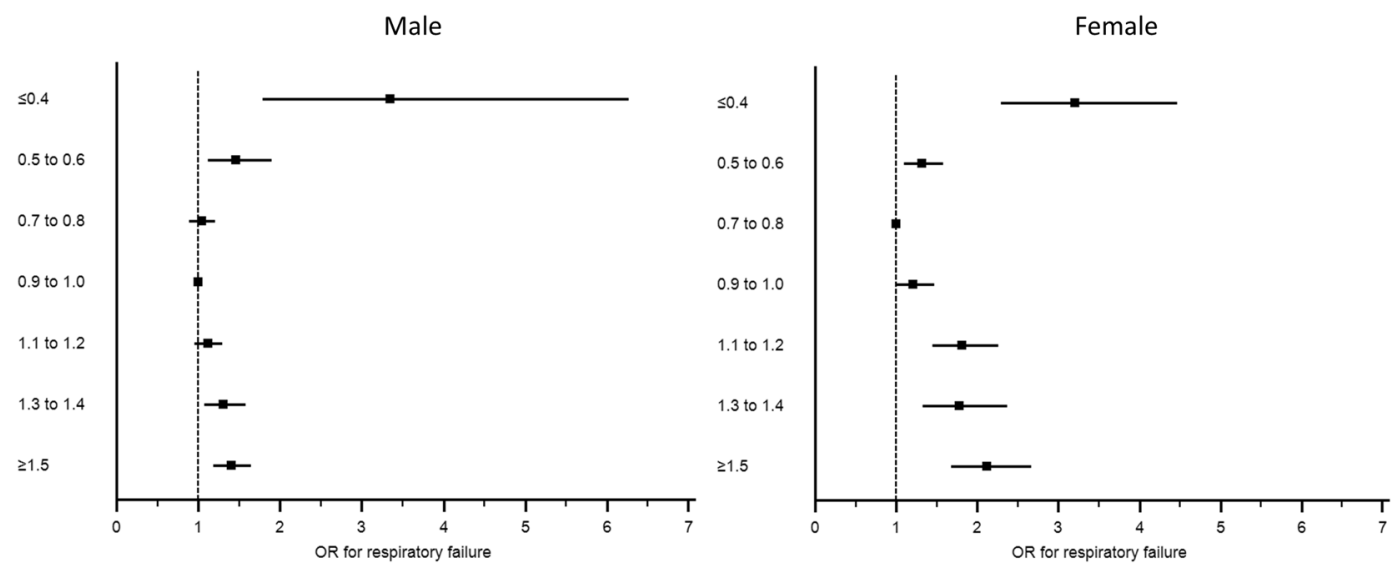

Figure 2. The association between admission serum creatinine and in-hospital respiratory failure requiring mechanical ventilation stratified by sex.

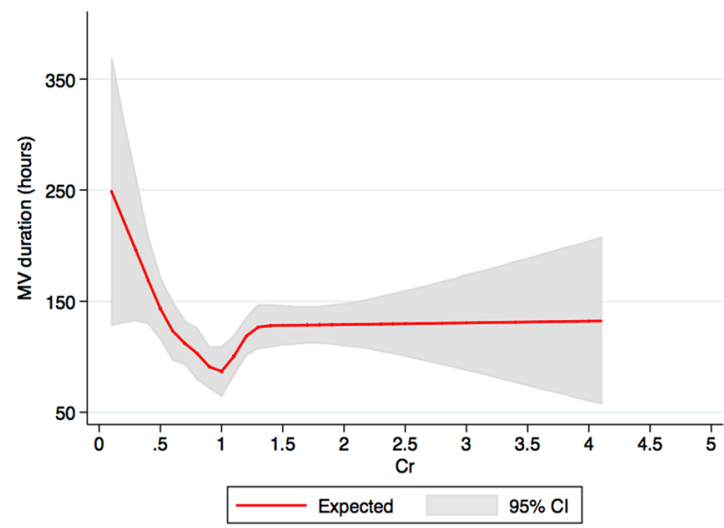

Figure 3. A restricted cubic spline showed the association between admission serum creatinine and the duration of mechanical ventilation among respiratory failure patients. Figure was created using STATA (StataCorp. 2015. Stata Statistical Software: Release 14. College Station, TX: StataCorp LP).

\begin{tabular}{|c|c|c|c|c|c|}
\hline \multirow[b]{2}{*}{$\begin{array}{l}\text { Serum creatinine level at } \\
\text { hospital admission }(\mathrm{mg} / \mathrm{dl})\end{array}$} & \multirow[b]{2}{*}{$\begin{array}{l}\text { Mechanical ventilation } \\
\text { duration (hours) }\end{array}$} & \multicolumn{2}{|l|}{ Univariate analysis } & \multicolumn{2}{|l|}{ Multivariate analysis } \\
\hline & & $\begin{array}{l}\text { Relative prolongation } \\
(95 \% \mathrm{CI})\end{array}$ & $\mathbf{p}$ & $\begin{array}{l}\text { Relative prolongation } \\
(95 \% \mathrm{CI})^{*}\end{array}$ & $\mathbf{P}$ \\
\hline$\leq 0.4$ & $79(36-241)$ & $1.70(1.19-2.44)$ & 0.004 & $1.44(1.02-2.07)$ & 0.04 \\
\hline $0.5-0.6$ & $56(25-141)$ & $1.28(1.03-1.58)$ & 0.03 & $1.25(0.99-1.57)$ & 0.06 \\
\hline $0.7-0.8$ & $41(19-112)$ & 1 (ref) & - & 1 (ref) & - \\
\hline $0.9-1.0$ & $41(18-94)$ & $0.89(0.74-1.07)$ & 0.20 & $0.97(0.80-1.17)$ & 0.75 \\
\hline $1.1-1.2$ & $44(20-118)$ & $1.04(0.86-1.26)$ & 0.66 & $1.16(0.96-1.41)$ & 0.13 \\
\hline $1.3-1.4$ & $51(21-119)$ & $1.06(0.85-1.32)$ & 0.62 & $1.17(0.93-1.48)$ & 0.19 \\
\hline$\geq 1.5$ & $51(21-122)$ & $1.18(0.98-1.42)$ & 0.08 & $1.23(1.01-1.51)$ & 0.04 \\
\hline
\end{tabular}

Table 4. The association between admission serum creatinine levels and duration of mechanical ventilation among respiratory failure patients. Adjusted for age, sex, race, BMI, principal diagnosis, Charlson Comorbidity Index, coronary artery disease, congestive heart failure, peripheral vascular disease, stroke, diabetes mellitus, chronic obstructive pulmonary disease, cirrhosis, hemi/paraplegia.

cubic spline in Fig. 3 demonstrated a non-linear association between admission serum creatinine and duration of mechanical ventilation. In adjusted analysis, very low admission SCr was associated with 1.44-time longer duration of mechanical ventilation, whereas markedly elevated admission SCr was associated with 1.23-time longer duration of mechanical ventilation, compared with admission SCr of $0.7-0.8 \mathrm{mg} / \mathrm{dL}$ (Table 4 ). In subgroup analysis based on sex, there was no significant association between admission serum creatinine and duration of mechanical duration in male patients but low admission serum creatinine $(\leq 0.6 \mathrm{mg} / \mathrm{dL})$ was significantly associated with longer duration of mechanical ventilation in female patients (Table S1). 


\section{Discussion}

Our findings revealed that either $\mathrm{SCr} \leq 0.6 \mathrm{mg} / \mathrm{dL}$ or $\geq 1.1 \mathrm{mg} / \mathrm{dL}$ at admission was significantly associated with a higher risk of respiratory failure requiring mechanical ventilation. Low $\mathrm{SCr}$ at admission $\leq 0.6 \mathrm{mg} / \mathrm{dL}$ in both men and women was significantly associated with a higher risk of respiratory failure requiring mechanical ventilation. Furthermore, low SCr at admission $\leq 0.4 \mathrm{mg} / \mathrm{dL}$ was significantly associated with longer duration of mechanical ventilation compared to admission level of SCr $0.7-0.8 \mathrm{mg} / \mathrm{dL}$.

To the best of our knowledge, we are the first group to report a lower SCr as an independent predictor for risk of respiratory failure requiring mechanical ventilation as well as an independent predictor for risk of prolonged duration of mechanical ventilation. We also depicted a U-curved association between SCr at admission and risk of respiratory failure requiring mechanical ventilation. Low SCr could reflect muscular wasting or sarcopenia and poor nutritional status ${ }^{12,13}$, which are strong risk factors for mechanical ventilation and a predictor of difficult weaning for mechanical ventilation as a reserved function of respiratory muscle and diaphragm among these patients is lesser than normal patients ${ }^{3,13,14}$. Moreover, malnutrition also results in weaker cellular mediated immunity and impaired surfactant production ${ }^{15,16}$. Even though volume overload could dilute SCr and result in lower levels ${ }^{17,18}$, we used SCr at admission before fluid resuscitation occurred and also adjusted for congestive heart failure to reduce the likelihood that fluid overload confounded the association. While high SCr could be due to acute kidney injury originated from the severity of primary illness, sepsis, or other causes ${ }^{19,20}$, all these conditions increase the risk of respiratory failure requiring mechanical ventilation as well.

Several limitations of this study are worth mentioning. This is a retrospective observational study so most likely there would be some residual confounders. Also, this is a single-center study with mostly White patients which may impact the generalizability of our data. Furthermore, we used only SCr at admission as a laboratory value without considering other laboratory values such as electrolytes or albumin, which might be potential confounders as well. However, there are several strengths to our study. We enrolled a large cohort of 67,045 patients to be able to adjust for several confounders which were specified a priori. Also, we used restricted cubic spline to visualize a potential non-linear association between SCr and risk of respiratory failure requiring mechanical ventilation. Last but not least, we also explored for effect modification by sex on this association.

In summary, low SCr level at admission is independently associated with increased in-hospital respiratory failure requiring mechanical ventilation. This association has been observed in patients of both sexes. Additionally, $\mathrm{SCr} \leq 0.4$ or $\geq 1.5 \mathrm{mg} / \mathrm{dL}$ were significantly associated with longer duration of mechanical ventilation compared to patients with SCr level at the admission of $0.7-0.8 \mathrm{mg} / \mathrm{dL}$.

Received: 21 September 2019; Accepted: 21 November 2019;

Published online: 10 December 2019

\section{References}

1. Andrews, R., Greenhaff, P., Curtis, S., Perry, A. \& Cowley, A. J. The effect of dietary creatine supplementation on skeletal muscle metabolism in congestive heart failure. Eur Heart J 19, 617-622 (1998).

2. Thongprayoon, C., Cheungpasitporn, W., Kittanamongkolchai, W., Harrison, A. M. \& Kashani, K. Prognostic Importance of Low Admission Serum Creatinine Concentration for Mortality in Hospitalized Patients. Am J Med 130, 545-554 e541, https://doi. org/10.1016/j.amjmed.2016.11.020 (2017).

3. Park, J. et al. Serum creatinine level, a surrogate of muscle mass, predicts mortality in peritoneal dialysis patients. Nephrol Dial Transplant 28, 2146-2155, https://doi.org/10.1093/ndt/gft213 (2013).

4. Schutte, J. E., Longhurst, J. C., Gaffney, F. A., Bastian, B. C. \& Blomqvist, C. G. Total plasma creatinine: an accurate measure of total striated muscle mass. J Appl Physiol Respir Environ Exerc Physiol 51, 762-766 (1981).

5. Pasala, S. \& Carmody, J. B. How to use... serum creatinine, cystatin C and GFR. Archives of disease in childhood - Education \& amp; practice edition 102, 37-43, https://doi.org/10.1136/archdischild-2016-311062 (2017).

6. Thongprayoon, C., Cheungpasitporn, W. \& Kashani, K. Serum creatinine level, a surrogate of muscle mass, predicts mortality in critically ill patients. J Thorac Dis 8, E305-311, https://doi.org/10.21037/jtd.2016.03.62 (2016).

7. Cartin-Ceba, R., Afessa, B. \& Gajic, O. Low baseline serum creatinine concentration predicts mortality in critically ill patients independent of body mass index. Crit Care Med 35, 2420-2423 (2007).

8. Scala, R. \& Heunks, L. Highlights in acute respiratory failure. European Respiratory Review 27, 180008, https://doi. org/10.1183/16000617.0008-2018 (2018).

9. Narendra, D. K. et al. Update in Management of Severe Hypoxemic Respiratory Failure. Chest 152, 867-879, https://doi. org/10.1016/j.chest.2017.06.039 (2017).

10. Gil, A. et al. Influence of mechanical ventilation on blood lactate in patients with acute respiratory failure. Intensive Care Med 24, 924-930, https://doi.org/10.1007/s001340050691 (1998).

11. Charlson, M., Szatrowski, T. P., Peterson, J. \& Gold, J. Validation of a combined comorbidity index. J Clin Epidemiol 47, 1245-1251 (1994).

12. Assy, N. et al. The changes in renal function after a single dose of intravenous furosemide in patients with compensated liver cirrhosis. BMC Gastroenterol 6, 39, https://doi.org/10.1186/1471-230x-6-39 (2006).

13. Thomas, M. E. et al. The definition of acute kidney injury and its use in practice. Kidney Int $\mathbf{8 7}, 62-73$, https://doi.org/10.1038/ ki.2014.328 (2015).

14. Kou, H. W. et al. Sarcopenia is an effective predictor of difficult-to-wean and mortality among critically ill surgical patients. PLoS One 14, e0220699, https://doi.org/10.1371/journal.pone.0220699 (2019).

15. Law, D. K., Dudrick, S. J. \& Abdou, N. I. Immunocompetence of patients with protein-calorie malnutrition. The effects of nutritional repletion. Ann Intern Med 79, 545-550, https://doi.org/10.7326/0003-4819-79-4-545 (1973).

16. Garbagni, R., Coppo, F., Grassini, G. \& Cardellino, G. Effects of lipide loading and fasting on pulmonary surfactant. Respiration 25, 458-464, https://doi.org/10.1159/000192580 (1968).

17. Lieu, C. \& Anderson, R. Serum creatinine: why lower may not be better. Crit Care Med 35, 2458-2459, https://doi.org/10.1097/01. ccm.0000284738.81354.fc (2007).

18. Thongprayoon, C. et al. The impact of fluid balance on diagnosis, staging and prediction of mortality in critically ill patients with acute kidney injury. Journal of nephrology 29, 221-227, https://doi.org/10.1007/s40620-015-0211-3 (2016).

19. Sime, F. B., Udy, A. A. \& Roberts, J. A. Augmented renal clearance in critically ill patients: etiology, definition and implications for beta-lactam dose optimization. Curr Opin Pharmacol 24, 1-6, https://doi.org/10.1016/j.coph.2015.06.002 (2015).

20. De Waele, J. J., Dumoulin, A., Janssen, A. \& Hoste, E. A. Epidemiology of augmented renal clearance in mixed ICU patients. Minerva Anestesiol 81, 1079-1085 (2015). 


\section{Author contributions}

C.T., W.C. and K.B.K. originated the idea, and designed study. C.T., W.C., A.C., S.T., and M.A.M. collected data. C.T. analyzed the data. C.T. and A.C. were responsible for writing the manuscript. W.C., S.T., M.A.M., K.B.K. supported the editing of the manuscript and added important comments to the manuscript. K.B.K. supervised the study. All authors had access to the data, read, and approved the final manuscript.

\section{Competing interests}

The authors declare no competing interests.

\section{Additional information}

Supplementary information is available for this paper at https://doi.org/10.1038/s41598-019-55362-w.

Correspondence and requests for materials should be addressed to K.B.K.

Reprints and permissions information is available at www.nature.com/reprints.

Publisher's note Springer Nature remains neutral with regard to jurisdictional claims in published maps and institutional affiliations.

(c) (i) Open Access This article is licensed under a Creative Commons Attribution 4.0 International

License, which permits use, sharing, adaptation, distribution and reproduction in any medium or format, as long as you give appropriate credit to the original author(s) and the source, provide a link to the Creative Commons license, and indicate if changes were made. The images or other third party material in this article are included in the article's Creative Commons license, unless indicated otherwise in a credit line to the material. If material is not included in the article's Creative Commons license and your intended use is not permitted by statutory regulation or exceeds the permitted use, you will need to obtain permission directly from the copyright holder. To view a copy of this license, visit http://creativecommons.org/licenses/by/4.0/.

(c) The Author(s) 2019 